Short Report

\title{
Presumptive diagnosis of malaria results in a significant risk of mis- treatment of children in urban Sahel
}

\begin{abstract}
Michael Olivar ${ }^{*}$, Michel Develoux ${ }^{2}$, Ari Chegou $\mathrm{Abari}^{3}$ and Louis Loutan ${ }^{1,4}{ }^{1}$ Department of Community Health, Tufts University School of Medicine, Boston, Massachusetts, USA; ${ }^{2}$ Laboratoire de Parasitologie, Faculté des Sciences de la Santé, Université de Niamey, Niamey, Niger; ${ }^{3}$ Circonscription Médicale de Bilma, Niger; ${ }^{4}$ Policlinique de Médecine, Hôpital Cantonal Universitaire de Genève, Geneva, Switzerland
\end{abstract}

Current health policies in most malaria-endemic countries, like Niger, adopt presumptive treatment with antimalarial drugs for management of cases of uncomplicated fever, especially among the child population. Given the great seasonal variations in incidence of malaria in Sahelian environments (GAZIN et al., 1988), it is essential to assess the relative importance of this disease at different seasons. Presumptive treatment during a season with very low transmission of malarial infection may result in a potentially high proportion of mis-diagnoses and the resulting mis-treatments.

In Niger, all suspected cases of malaria are systematically treated with a course of antimalarial drugs, without establishing a parasitological diagnosis. The National Malaria Control Programme of Niger lays down that all non-complicated simple fevers (without any obvious source) should be considered first as malarious.

To assess the efficacy of this policy, 2 groups of children aged 1 to 5 years were selected from 5 randomly-chosen mother-and-child health clinics in the capital city, Niamey, at the end of one dry season (May and June 1989) and at the end of a rainy season (September and October 1989). All newcomers with a body temperature ascertained in the clinic to be above $38^{\circ} \mathrm{C}$ were evaluated during the duration of the study. Patients with seizures or unconscious at the time of the visit were not included, since they are immediately referred to the hospital.

After being seen by the clinic personnel, who established a diagnosis and prescribed treatment in their usual fashion, children underwent a more thorough physical examination and thick and thin blood films were prepared.

Blood films were stained with Giemsa and 200 high-power fields were examined. Based on the work of BOUDON et al. (1988), who established a 'pyrogenic threshold' for falciparum malaria in West Africa at 10000 infected erythrocytes per $\mu l$, the criteria used

"At the time of the study, Dr Olivar was in le Faculté des Sciences de la Santé, Université de Niamey (bilateral programme between Tufts University and l'Université de Niamey).

Address for correspondence: Dr Michael Olivar, Department of Community Health, Tufts University School of Medicine, 136 Harrison Avenue, Boston, MA 02111, USA.

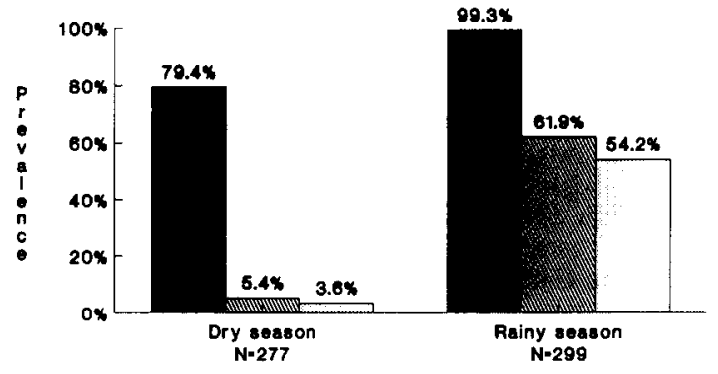

Figure. Prevalences of malaria diagnoses, infections and clinical cases in Niamey, Niger, 1989. Solid bars: presumptive diagnoses; hatched bars: malaria infection; stippled bars: clinical cases. The differences between all pairs of values for the two seasons are significant $(P<0-0001)$.

in this study to confirm a diagnosis of a malaria attack were (i) parasitaemia greater than 10000 infected cells/ $\mu \mathrm{l}$, (ii) a positive blood film from patients who had received an effective dose of antimalarial drug before clinic attendance (since self-medication was common), and (iii) a positive blood film without any other obvious source of fever from persons who had received insufficient prior antimalarial therapy.

Five hundred and seventy-six children were studied, 277 in the dry season and 299 in the rainy season. Parasite rates were $15 / 277(5.4 \%)$ in the dry season and $185 / 299(61.9 \%)$ during the rainy season. Plasmodium falciparum was found in 199/200 positive cases $(99.5 \%)$, mixed with $P$. ovale in $3.5 \%$ and with $P$. malariae in $3.5 \% ; P$. ovale was found alone in one case $(0.5 \%)$. The gametocyte index of the total sample was $9.5 \%$, and in 4 cases $(2 \%)$ only asexual forms were found. These cases were excluded from the parasite count.

According to our diagnostic criteria 172 children $(87.8 \%$ of those with asexual stages) of the total samples had a malaria attack: $10 / 277$ children (3.6\%) in the dry season and $162 / 299(54.2 \%)$ during the rainy season $(P<0.0001)$. A diagnosis of malaria was established by the clinic personnel in 220/277 children $(79 \cdot 4 \%)$ during the dry season and $297 / 299(99 \cdot 3 \%)$ in the rainy season (Figure). Therefore, during the dry season only $10 / 220$ malaria cases $(4 \cdot 5 \%)$ identified by the health personnel fulfilled the study criteria for a malaria attack, whereas during the rainy season $160 / 297(53.9 \%)$ of the clinic's diagnoses were correct according to the same criteria.

Our data revealed that, even in the high period of transmission (end of the rainy season), almost half of the presumptive diagnoses established by clinic personnel were erroneous, according to our criteria: $137 / 297(46 \cdot 1 \%)$. Furthermore, during the period of low transmission, because of the very low morbidity due to malaria, the rate of erroneous diagnoses was extreme: $210 / 220(95.5 \%)$, significantly higher than in the rainy season $(P<0.0001)$. GAYE et al. (1989) found similar rates of mis-diagnosis in Dakar during the rainy season. However, their study did not include the dry season.

A precise diagnosis of malaria is very difficult to establish and has to take into account a multiplicity of factors. TRAPE et al. (1985) showed, in the Congo, the complexity of making a clinical diagnosis of malaria. In the criteria we used we considered prior intake of 
antimalarial drugs, intercurrent infections, and parasite density. We are aware of the difficulty of applying these criteria as a general rule; however, the great variation in morbidity due to malaria at different seasons raises questions about the validity of presumptive diagnosis of malaria in febrile children from an urban environment in an area where transmission is very seasonal. Therefore, we think that in the Sahelian context the currently recommended practice of presumptive treatment should be reviewed, especially during the season of low transmission. It produces an excessive over-estimation of malaria cases which precludes investigation of other possible causes of fever, and the prescription of antimalarial treatment with no justification when other treatments may be required. Failure to consider other diagnoses in such a high proportion of patients may increase the mortality rate of children under 5 years old.

\section{Acknowledgements}

This study was partly funded by Band Aid/Live Aid, through an epidemiology training project. We thank $\mathrm{Dr}$
Alain Prual for his valuable suggestions and comments on the manuscript.

\section{References}

Baudon, D., Gazin, P., Galaup, B., Pellotier-Guinart, E. \& Picq, J. J. (1988). Fiabilité de l'examen clinique dans le diagnostic des fièvres palustres en zone d'endémie Ouest-Africaine. Médecine Tropicale, 48, 123-126.

Gaye, O., Bah, I. B., Bengue, I. B., Diallo, S. \& Faye, O. (1989). Morbidité palustre en milieu urbain. Etude de 353 accès fébriles. Médecine Tropicale, 49, 401-404.

Gazin, P., Robert, V., Cot, M., Simon, J., Halma, J. M., Darriet, F., Legrand, D., Carnevale, P. \& AmbroiseThomas, P. (1988). Le paludisme dans l'Oudalan, région sahélienne du Burkina-Faso. Annales de la Société Belge de Médecine Tropicale, 68, 255-264.

Trape, J. F., Peelman, P. \& Morault-Peelman, B. (1985). Criteria for diagnosing clinical malaria among a semiimmune population exposed to intense and perennial transmission. Transactions of the Royal Society of Tropical Medicine and Hygiene, 79, 435-442.

Received 7 February 1991; revised 8 May 1991; accepted for publication I6 May 1991

\section{Announcement}

\section{Health and population data for developing countries available through USAID project}

The Demographica and Health Survey (DHS) Program, funded by the U.S. Agency for International Development (USAID), is contributing unique data to the global database on population and health. To date, over thirty-five surveys have been conducted in developing countries including Botswana, Kenya, Nigeria, Senegal, Zimbabwe, Indonesia, Sri Lanka, Peru, Bolivia, Ecuador, Egypt and Morocco.

The surveys address topics such as infant and child mortality, prenatal and delivery care, fertility and family planning, breastfeeding, immunization, diarrhea, acute respiratory infection, fever, child nutritional status, and women's and husband's background and employment. Some surveys also gather information on causes of child death, maternal mortality, knowledge of AIDS, social marketing and availablility of services.

In general, DHS datasets are available to researchers after the publication of the country survey report. For a few surveys, written permission is necessary from the in-country survey organization. The data files are accompanied by questionnaires, machine-readable descriptions and associated documentation. Currently, the cost of a DHS dataset is $\$ 200$ ( $\$ 50$ for researchers in developing countries).

To order a DHS dataset, send a completed request form and description of your analysis project to the DHS Data Archive. Request forms are available from the archive. The address is:

DHS Data Archive

Institute for Resource Development

8850 Sanford Boulevard

Columbia, Maryland 21045, USA

Telephone: (301) 290-2977

Telex: 87775 